\title{
On the Effects of Soil Properties on Leak Noise Propagation in Plastic Water Distribution Pipes
}

\author{
M.J. Brennan ${ }^{a}$, M. Karimi ${ }^{b}$, J.M. Muggleton ${ }^{c}$, F.C.L Almeida ${ }^{d}$, F. Kroll de Lima ${ }^{a}$, P.C. \\ Ayala $^{a}$, D. Obata ${ }^{a}$, A.T. Paschoalini ${ }^{a}$, N. Kessissoglou ${ }^{b}$ \\ a Department of Mechanical Engineering, UNESP, Ilha Solteira, São Paulo, 15385-000, Brazil \\ b School of Mechanical and Manufacturing Engineering, University of New South Wales, \\ Australia, Sydney, NSW 2052, Australia \\ c Institute of Sound and Vibration Research, University of Southampton, United Kingdom \\ ${ }^{\mathrm{d}}$ Department of Biosystems Engineering, UNESP, Tupã, São Paulo, 17602-496, Brazil \\ * Corresponding author
}

No of pages: 26

No of figures: 10

No of tables: 4

21 September 2017

Revised 20 February 2018 


\begin{abstract}
In many countries, leaks are located in water distribution pipes by using the cross-correlation of pipe vibration measured either side of a suspected leak. However, in modern plastic pipes this can be problematic due to strong coupling between the water, the pipe and the soil, affecting the propagation of leak noise within the pipe. This paper concerns an analytical, numerical and experimental investigation into the way in which soil properties influence leak noise propagation in buried plastic water pipes. The analytical model allows a detailed investigation into the physical effects of the soil on leak noise (wave) propagation in the pipe, in particular on the wave speed and wave attenuation. Results highlight that, in addition to the pipe hoop stiffness, the shear stiffness of the soil can have a significant effect on the wave speed in the pipe. Experimental measurements were conducted at two different sites - one in the UK and the other in Brazil. In the UK system, both dilatational and shear waves in the soil propagate away from the pipe, resulting in large wave attenuation in the pipe. However, in the Brazil system, only shear waves propagate resulting in smaller wave attenuation in the pipe.
\end{abstract}

Keywords: water leak detection; wave propagation; pipe vibro-acoustics 


\section{Introduction}

Water leakage is a serious problem in many countries. It is estimated that 40 to $50 \%$ of drinking water is wasted through leakage in developing countries, and less than $10 \%$ in countries where the utilities are well-maintained, such as Japan [1,2]. To determine if a leak is present in a specific part of the network, pressure measurements together with flow measurements are often used [3]. Leak noise correlators can then be used to determine a more precise location of the leak [4]. Although correlators work well for metallic pipes, their performance with plastic pipes is more limited [5-7]. Two main factors affect correlator performance in this case. They are the relatively high rates of attenuation experienced by the leak noise propagating along the pipes and the variability in the speed (wave-speed) at which it propagates. The wave-speed can be heavily influenced by the pipe properties and the surrounding soil [8-13]. Thus, the maximum distance between the sensors on either side of the leak is limited by the attenuation of the leak noise. Further, the accuracy with which the leak can be located is related to the accuracy of the wave-speed estimate. In nearly all cases, the wave-speed is estimated from an empirical database determined from calculations made using assumed material properties and pipe geometry.

Motivated by differing measurements of pipe wave speed and attenuation in two different sites, one in the UK and one in Brazil [14], the aim of this paper is to determine the way in which soil properties affect the characteristics of the pipe wave responsible for leak noise propagation. The investigation focuses on the factors that affect wave-speed and wave attenuation. It includes analytical modelling of wave propagation in a buried water pipe, development of a numerical model based on the finite element method whereby the water, the pipe and the surrounding soil comprise a fully coupled three-part system, and experimental measurements 
of pipe wave speed and attenuation at two different sites, one of which is in the UK and the other is in Brazil. The analytical model is formulated in terms of the dynamic stiffness of the pipe and the soil, which facilitates physical insight into the effects of soil parameters on leak noise propagation.

The paper is organised as follows. Following this introduction, analytical and finite element models of a generic pipe system are described in Section 2. Following validation of the analytical model in Section 3, a detailed investigation into the physical effects of the soil on wave propagation in the pipe is then carried out in Section 4. It also describes a parametric study. Finally, some conclusions are drawn in Section 5.

\section{Modelling of the pipe system}

Two models of the pipe system are described in this section, namely an analytical model and a finite element model. The analytical model is developed to allow a detailed analysis to be conducted into the way in which the soil properties affect the propagation of leak noise. However, because this model contains some simplifying assumptions, a finite element model is also developed. This model has fewer simplifying assumptions and so it can be used to help validate the analytical model. Moreover, it can be used to readily give a picture of the wave propagation in the soil.

\subsection{Analytical Model}

Several analytical models of a buried water pipe system have been developed in the literature, with differing levels of complexity, for example. $[8,10,12]$, but the simplest one that contains the essential physics is believed to be the one in reference [10]. In this model, it is assumed that the pipe only connects dynamically with the soil in the radial direction, so there is no excitation 
of the soil in the axial direction of the pipe. Although this assumption is not strictly justified from the physical point of view, it does significantly simplify the model and facilitates physical interpretation of the effects of the soil on leak noise propagation in the pipe. In reference [12] it is shown that the inclusion of a no-slip condition between the pipe and the soil has only a marginal effect on the wave propagation properties in the frequency range considered in this paper $(0-600 \mathrm{~Hz})$. Moreover, in the numerical model described in the next section, no simplifying assumptions are made about the pipe-soil face interface, and the results are found to be comparable with the analytical model for the cases studied. Thus, for the purposes of this paper, a slip condition between the pipe and the surrounding soil is assumed.

A schematic diagram of a buried plastic water pipe is shown in Fig. 1. It has a mean radius $a$ and pipe-wall thickness $h$, with density $\rho$ and complex Young's modulus $E^{*}=E\left(1+\mathrm{j} \eta_{p}\right)$ in which $E$ is the storage modulus and $\eta_{p}$ is the loss factor. The pipe contains water with bulk modulus $B_{\text {water }}$, and is buried in soil with density $\rho_{\text {soil }}$, and bulk modulus $B_{\text {soil }}^{*}=B_{\text {soil }}\left(1+\mathrm{j} \eta_{d}\right)$, where $B_{\text {soil }}$ is the storage modulus and $\eta_{d}$ is the compressional loss factor; the shear modulus is given by $G^{*}=G\left(1+\mathrm{j} \eta_{s}\right)$, where $G$ is the storage modulus and $\eta_{s}$ is the shear loss factor. The approximate complex wavenumber related to leak noise propagation is given in [10], which is written here in the following form to aid physical insight

$$
k=k_{\text {water }}\left(1+\frac{K_{\text {water }}}{K_{\text {pipe }}+K_{\text {soil }}}\right)^{\frac{1}{2}} \text {, }
$$

where $k_{\text {water }}=\omega / c_{\text {water }}$ is the wavenumber of the water within the pipe, in which $c_{\text {water }}=1500 \mathrm{~m} / \mathrm{s}$ is the speed of sound in water; $K_{\text {water }}=2 B_{\text {water }} / a$ is the dynamic stiffness 
(pressure/displacement) of the water in the pipe, $K_{\text {pipe }}=E^{*} h / a^{2}-\rho h \omega^{2}$ is the dynamic stiffness of the pipe-wall, and $K_{\text {soil }}$ is the dynamic stiffness of the surrounding soil. The dynamic stiffness of the soil can be written in terms of three components, so that $K_{\text {soil }}=K_{1}+K_{2}+K_{3}$, in which [14]

$$
\begin{gathered}
K_{1}=\left(B_{\text {soil }}^{*}-\frac{2 G^{*}}{3}\right) \frac{k_{d}^{2}}{k_{d}^{r}}\left(1-2 \frac{k^{2}}{k_{r}^{2}}\right) \frac{H_{0}\left(k_{d}^{r} a\right)}{H_{0}^{\prime}\left(k_{d}^{r} a\right)}, \\
K_{2}=-2 G^{*} k_{d}^{r}\left(1-2 \frac{k^{2}}{k_{r}^{2}}\right) \frac{H_{1}^{\prime}\left(k_{d}^{r} a\right)}{H_{1}\left(k_{d}^{r} a\right)}, \\
K_{3}=-4 G^{*} k_{r}^{r} \frac{k^{2}}{k_{r}^{2}} \frac{H_{1}^{\prime}\left(k_{r}^{r} a\right)}{H_{1}\left(k_{r}^{r} a\right)} .
\end{gathered}
$$

where $k_{d}^{r}=\sqrt{k_{d}^{2}-k^{2}}$ and $k_{r}^{r}=\sqrt{k_{r}^{2}-k^{2}}$ are the soil radial wavenumbers, in which $k_{d}$ and $k_{r}$ are the dilatational and shear wavenumbers in the soil, respectively; $H_{0}(\bullet)$ and $H_{1}(\bullet)$ are Hankel functions of the second kind, and ' denotes a spatial derivative. Note, that if the shear stiffness is set to zero, then $K_{1}$ is the dynamic stiffness of a dilatational wave into an acoustic medium such as water. The other two terms, $K_{2}$ and $K_{3}$, are clearly zero in this case. The behaviour of the terms comprising $K_{\text {soil }}$ and their effects on wave propagation in the pipe are discussed in detail in Section 4. The attenuation of the wave in the pipe in terms of $\mathrm{dB} / \mathrm{m}$ is given by [8] 


$$
\text { Wave attenuation }(\mathrm{dB} / \mathrm{m})=-\frac{20 \operatorname{Im}\{k\}}{\ln (10)}
$$

\subsection{Finite Element Model}

The pipe system shown in Fig. 1 is modelled as a three-part system comprising water, the pipe and the surrounding soil. A soil medium of infinite extent is used for simplicity and to reduce the simulation calculation time. Pipe fittings and joints are neglected. A schematic diagram of the numerical model is shown in Fig. 2. A 2D axisymmetric finite element model of a waterpipe-soil system is developed using the commercial software COMSOL Multiphysics (v5.2). The water-pipe-soil section is axis-symmetric and a monopole source is placed at the centre of the pipe to excite a predominantly fluid wave in the pipe, corresponding to the wavenumber $k$ given by Eq. (1). A perfectly matched layer (PML) is applied on the boundary of the computational domain to simulate an infinite medium.

A fine mesh of triangular elements is used in the discretisation of the model to ensure accurate numerical predictions. The largest element size is less than $1 / 6$ the wavelength at the highest frequency considered $(600 \mathrm{~Hz})$. The water is modelled as a fluid (lossless acoustic medium) using the COMSOL Pressure Acoustics module, and the pipe and the surrounding soil are modelled as linear elastic media using the Solid Mechanics module. The Acoustic-Structure boundary condition is used to couple the pressure acoustics model (water) to the structural component (pipe). The numerical computation is performed in the frequency domain to obtain the frequency response function (FRF) of the water-pipe-soil system between the monopole source excitation and the pressure at various positions either in the pipe, radial displacements on the pipe-wall, and displacements in the soil. 
To determine the leak noise propagation characteristics, such as the wave-speed and the wave attenuation, the FRFs between the acoustic source and the radial displacement of the pipe at two arbitrary positions $A$ and $B$ along the length of the pipe, $D_{A}(\omega)$ and $D_{B}(\omega)$, are first calculated. The transmissibility between the radial pipe displacements $W_{A}(\omega)$ and $W_{B}(\omega)$ corresponding to these positions is given by,

$$
T_{A B}(\omega)=\frac{D_{B}(\omega)}{D_{A}(\omega)}=\frac{W_{B}(\omega)}{W_{A}(\omega)}
$$

The real part of the wavenumber, which is related to the wave-speed $c$ by $\operatorname{Re}\{k(\omega)\}=\omega / c$, can be determined from the phase of $T_{A B}(\omega)$. This is because the phase between positions $A$ and $B$ is given by $\phi_{A B}(\omega)=-\operatorname{Re}\{k(\omega)\} d$, where $d$ is the distance between $A$ and $B$. Thus,

$$
\operatorname{Re}\{k(\omega)\}=\frac{-\phi_{A B}(\omega)}{d}
$$

In practice, plastic water pipe systems tend to exhibit broadly non-dispersive like behaviour over the frequency range in which measurements are made [9] (this is discussed further in Section 4). Thus, the wave speed is approximately constant, so the phase, and hence the real part of the wavenumber, tend to have approximately straight-line behaviour as a function of frequency. The attenuation of the wave as a function of frequency, in terms of $\mathrm{dB} / \mathrm{m}$, is given by

$$
\text { Wave Attenuation }(\mathrm{dB} / \mathrm{m})=\frac{-20 \log _{10}\left(T_{A B}(\omega)\right)}{d}
$$




\section{Experimental Work}

Experimental measurements of pipe wave-speed and wave attenuation were carried out at two different sites, one of which is in the UK [9] and the other is in Brazil. The nominal properties of the pipe and soil are given in Tabs. 1 and 2 respectively. The soil type corresponding to the UK system is soil type I, which is representative of much of the soil found in the UK. For the Brazil system, the soil is type II, which is representative of the soil in São Paulo.

The measurements for the UK system are described in detail in [9] and so are only briefly described here. The layout is shown in Fig. 3(a). It consists of a 34 metre long test rig, in which the pipe was not connected to a pressurised system, and so the static pressure was due to the $1.5 \mathrm{~m}$ head of water in the termination tanks. The predominantly fluid wave was excited by modified underwater loudspeaker using a stepped sine signal in the frequency range of $30 \mathrm{~Hz}$ to $1 \mathrm{kHz}$ with $1 \mathrm{~Hz}$ increments. The dynamic pressure was measured using two hydrophones positioned 2 metres apart.

The measurements for the Brazil system were conducted using a test rig that had a smaller diameter pipe than the UK system and a much shorter length of pipe. The layout of this test rig is shown in Fig. 3(b). The pipe was pressurised with a centrifugal pump to a pressure of about 3.4 bar. The predominantly fluid wave was excited by opening a valve to generate broadband noise. Signals related to this wave were measured using accelerometers at two access points, 7 metres apart, downstream of the noise source. The accelerometers were able to sense the leak noise because of the strong coupling between the fluid and the pipe wall. 
The real part of the wavenumber was calculated from the measured data in both cases using Eqs. (4) and (5), and the wave attenuation using Eq. (6). The results are plotted in Fig. 4. together with the predictions from the analytical and numerical models.

For comparison of the analytical and numerical predictions with the experimental results, the system parameters have to be determined as the inputs to the analytical and numerical models. The plastic pipes at both sites are made from the same material, but the Brazil system has a pipe of smaller radius and thickness compared to the UK system. The pipe geometry and material properties were given by the pipe specifications and are listed in Tab. 1 . The pipe loss factor was taken from [10]. For the UK system, nominal soil properties corresponding to the bulk and shear moduli were also taken from [10]. However, as discussed later in Section 4, the shear modulus of the soil has a dominant effect on the wave speed and hence the real part of the wavenumber. This is because it creates a stiffness effect of the soil on the pipe and restricts its radial motion, thereby increasing the speed at which leak noise propagates. To obtain a better fit between the predicted and measured real part of the wavenumber for the Brazil pipe system shown in Fig. 4(bi), the shear modulus of soil type II was increased from $1.8 \times 10^{8} \mathrm{~N} / \mathrm{m}^{2}$ which was given in [10] to $2.41 \times 10^{8} \mathrm{~N} / \mathrm{m}^{2}$. To achieve a good fit for the losses in the Brazil pipe system, which is expressed as wave attenuation in $\mathrm{dB} / \mathrm{m}$, a loss factor of 0.15 was chosen for the shear modulus. For consistency, this value was also chosen for the losses associated with the bulk modulus, but this was found to have a negligibly small effect on the results. Also, for the sake of consistency, a value of 0.15 was chosen for the loss factors associated with the shear and bulk moduli for the UK pipe test rig, but this was also found to have a negligibly small effect. The effects of the soil properties on leak noise propagation along the pipe is discussed in detail in Section 4. 
Examination of Fig. 4. shows that the predictions from the analytical and numerical models, and the experimental results agree well. The deviations in the experimental data from the predicted results, especially at low frequencies, are thought to be due to the effect of the free surface of the ground, and reflections of the predominantly fluid wave due to the finite length of the pipe. These effects are not captured in either the analytical or the numerical model because of the simplifying assumptions.

The two fundamental differences between the two pipe systems considered here are the wave speeds which were calculated using Eq. (1). They were found to be approximately $376 \mathrm{~m} / \mathrm{s}$ for the UK system and $544 \mathrm{~m} / \mathrm{s}$ for the Brazil system. Moreover, there was much higher attenuation for the UK system for frequencies greater than around $200 \mathrm{~Hz}$. Although this is partly due to the pipe geometry, it is largely due to the much higher shear stiffness of the Brazilian soil. This is discussed in more detail in the following section.

\section{Analysis}

\subsection{Dynamic stiffness and wave-speed}

The analysis conducted in this section is centred around the dynamic stiffness of the pipe, $K_{\text {pipe }}$, and the dynamic stiffness of the soil, $K_{\text {soil }}=K_{1}+K_{2}+K_{3}$, in which the three terms are given in Eqs. (2a-c). Before conducting this analysis, however, it is instructive to briefly examine the base-line result corresponding to a water-filled massless plastic pipe in air. In this case $K_{\text {soil }}=0$, and Eq. (1) reduces to the Korteweg equation given by $k=k_{\text {water }}\left(1+K_{\text {water }} / K_{\text {pipe }}\right)^{\frac{1}{2}}$. It should be noted that the ratio of the fluid stiffness, $K_{\text {water }}=2 B_{\text {water }} / a$, to the static hoop stiffness of the pipe, $K_{\text {pipe }}=E h / a^{2}$, is about 22 for the UK pipe and about 30 for the Brazil pipe, and this difference is the principal reason why the predominantly fluid wave speed in 
both pipe systems are much lower than the wave speed in water $(1500 \mathrm{~m} / \mathrm{s})$. It should also be noted that the Korteweg wave is non-dispersive. If the mass of the pipe is taken into account, then the real part of its dynamic stiffness is given by $\operatorname{Re}\left\{K_{\text {pipe }}\right\}=E h / a^{2}-\rho h \omega^{2}$. Thus, the effective stiffness of the pipe reduces with frequency, causing the wave to become dispersive, with the wave speed reducing marginally within the frequency range $0-600 \mathrm{~Hz}$.

The terms that comprise the soil dynamic stiffness are given in Eqs. (2a-c). appear to be relatively complicated because of the combinations of Hankel functions that they contain, and this causes difficulty in gaining physical insight. Unfortunately, these Hankel functions cannot be simplified in all cases in the frequency range of interest, because small or large arguments are not always valid. However, because the terms represent the dynamic stiffness effects of the various waves generated in the soil by the pipe vibration, it is relatively straightforward to interpret their effects. The first term of the soil stiffness, $K_{1}$, relates to a dilatational wave, but it is influenced by both the shear and bulk moduli. The terms $K_{2}$ and $K_{3}$ only exist if there is a shear stiffness in the external medium. Hence these two terms are summed for convenience and termed a shear related wave, even though the arguments for the Hankel functions in $K_{2}$ are related to the dilatational wave number and the arguments for the Hankel functions in $K_{3}$ are related to the shear wave number. Further, when the wavelengths of the dilatational and shear waves in the soil are much greater that the diameter of the pipe, then $\operatorname{Re}\left\{K_{2}+K_{3}\right\} \approx 2 G / a$, i.e., is simply proportional to the stiffness of the soil [10].

To make the results easier to interpret, the dynamic stiffnesses are normalised by the static hoop stiffness of the pipe, $E h / a^{2}$ so that $\hat{K}_{\text {pipe }}=K_{\text {pipe }} /\left(E h / a^{2}\right), \hat{K}_{i}=K_{i} /\left(E h / a^{2}\right)$, where $i=1$ 3. The soil dynamic stiffness components have a stiffness (mass) effect on the pipe when the 
real part of the dynamic stiffness is positive (negative). As the terms are normalised by the hoop stiffness of the pipe, then a value of the normalised real part of the soil dynamic stiffness greater (smaller) than unity means that the soil has a greater (lesser) effect than that of the pipe. The imaginary part of the soil dynamic stiffness is related to the energy that is lost from the pipe, which is either due to damping in the pipe-wall, damping in the soil or wave radiation into the soil. If the sum of the imaginary parts of the soil dynamic stiffness is divided by the sum of the real parts of the soil dynamic stiffness then an effective loss factor for the soil can be calculated. Note that this is not the same as the material loss factor of the soil.

The real and imaginary parts of the normalised component dynamic stiffnesses, $\hat{K}_{\text {pipe }}, \hat{K}_{1}$ and $\hat{K}_{2}+\hat{K}_{3}$, as well as $\hat{K}_{\text {total }}=\hat{K}_{\text {pipe }}+\hat{K}_{1}+\hat{K}_{2}+\hat{K}_{3}$, are shown in Figs 5(ai, aii) and (bi, bii) for the UK pipe test rig, and the Brazil pipe test rig respectively. First, examining the real part of the dynamic stiffness for the UK pipe test rig (Fig. 5(ai)), it can be seen that the effect due to the mass of the pipe-wall is very small. It can also be seen that $\operatorname{Re}\left\{\hat{K}_{1}\right\}$, corresponding to the dilatational wave, has a mass-loading effect on the pipe, but this effect is also very small. The $\operatorname{Re}\left\{\hat{K}_{2}+\hat{K}_{3}\right\}$, corresponding to the shear related wave, is stiffness-like and is roughly constant, but its value is small compared to the normalized dynamic hoop stiffness of the pipe, $\operatorname{Re}\left\{\hat{K}_{\text {pipe }}\right\}$ . As $\operatorname{Re}\left\{\hat{K}_{\text {total }}\right\}$ is approximately constant in the frequency range $(0-600 \mathrm{~Hz})$, this implies that the wave speed will also be approximately constant. If the corresponding graph is examined for the Brazil pipe test rig, which is in Fig. 5(bi), it can be seen that the main difference is that the $\operatorname{Re}\left\{\hat{K}_{2}+\hat{K}_{3}\right\}$ is much larger, being about 2.5 times larger than the normalized dynamic hoop stiffness of the pipe $\operatorname{Re}\left\{\hat{K}_{\text {pipe }}\right\}$. Comparing Figs. 5(ai) and 5(bi) reveals that the increase 
of almost two orders of magnitude in the bulk modulus $B_{s}$ for the Brazil pipe test rig with soil type II compared to the UK pipe test rig with soil type I, has practically no effect on the dilatational wave represented by $\operatorname{Re}\left\{\hat{K}_{1}\right\}$. This is because the dilatational wave has only a mass-loading effect on the pipe in this case, and so is not affected by the bulk stiffness of the soil.

Using Eq. (1), the wave speeds for the UK and Brazil pipe test rigs are plotted in Figs. 6(a) and (b), respectively. It can be seen that the wave speeds do not vary much over the frequency range of interest. An approximate expression for the wave speed can be determined from Eq. (1) by noting that the mass of the pipe can be neglected together with the mass-loading effects due to the dilatational wave. Furthermore, using the approximation $\operatorname{Re}\left\{K_{2}+K_{3}\right\} \approx 2 G / a$, a simplified expression for the wave-speed in the pipe can be obtained as,

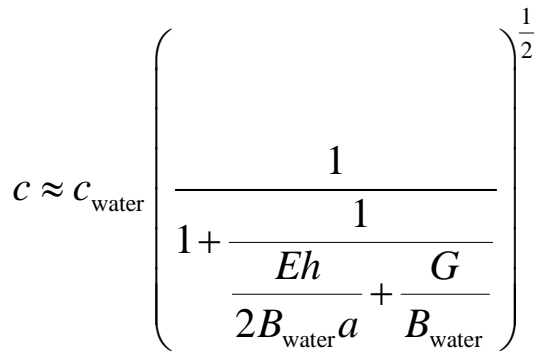

The wave speed approximated using Eq. (7) is also plotted in Fig. 6 and is shown to give a reasonable estimate in both cases. The way in which the shear stiffness affects the wave speed is evident. The wave-speed for the water-filled pipe in air is also plotted in Figs. 6(a) and (b). It is higher for the UK pipe than for the Brazil pipe, which is due to a higher hoop stiffness in the UK pipe. However, the shear stiffness of soil type II has a profound effect on the wave 
speed in the Brazil pipe, as shown in Fig. 6(b), increasing it dramatically compared to the buried UK pipe whose wave speed is only marginally affected by the soil.

The difference in the wave speeds in the soil and the pipe can have a profound effect on the wave attenuation in the pipe, as they determine whether or not a wave will propagate away from the pipe into the soil and hence appear as energy loss to the pipe. The wave speeds corresponding to the dilatational wave $c_{d}$, and the shear wave $c_{s}$, are respectively given by,

$$
c_{d}=\sqrt{\left(B_{\text {soil }}+4 G / 3\right) / \rho_{\text {soil }}}, \quad \quad C_{s}=\sqrt{G / \rho_{\text {soil }}} \text {. }
$$

The values of the wave speeds for the UK and Brazil pipe test rig systems are given in Tab. 3 , together with the angles at which the waves, which have a conical shape, propagate away from the pipe. The angles are determined by $\theta_{d, s}=\cos ^{-1}\left(c / c_{d, s}\right)$, where $c$ is calculated using Eq. (7). If the wave-speed in the pipe is less than the wave-speed in the soil, then that wave does not propagate away from the pipe. An exponential decaying wavefield then exists in the soil in the radial direction, with a wave propagating in the direction of the pipe. If the wave speed in the pipe is greater than the wave speed in the soil, then a wave propagates away, extracting energy from the pipe. From Tab. 3, it can be seen that in the UK pipe test rig both dilatational and shear waves propagate away from the pipe, whereas in the Brazil pipe test rig, only the shear wave propagates away. This is important from the point of view of wave attenuation in the pipe.

\subsection{Dynamic stiffness and wave attenuation}


Consider now the imaginary parts of the dynamic stiffness of the pipe and soil for the UK pipe test rig shown in Fig. 5(aii). The normalised imaginary part of the dynamic stiffness of the pipe is simply given by the loss factor of the pipe, i.e., $\operatorname{Im}\left\{\hat{K}_{\text {pipe }}\right\}=\eta_{p}=0.06$. There are no simple expressions for $\operatorname{Im}\left\{\hat{K}_{1}\right\}$ corresponding to the dilatational waves and $\operatorname{Im}\left\{\hat{K}_{2}+\hat{K}_{3}\right\}$ related to the shear stiffness of the soil, but it can be seen that both of these have similar behaviour, with $\operatorname{Im}\left\{\hat{K}_{2}+\hat{K}_{3}\right\}$ being slightly larger than $\operatorname{Im}\left\{\hat{K}_{1}\right\}$. It can also be seen that above about $250 \mathrm{~Hz}$, the loss through these effects is greater than the loss within the pipe wall (due to the pipe loss factor). The imaginary parts of the dynamic stiffness for the Brazil pipe exhibit different behaviour. Examining Fig. 5(bii), it is clear that the loss due to the pipe-wall is similar, but the loss due to the dilatational wave is practically zero ${ }^{1}$, because this wave does not propagate away from the pipe, and only has a mass loading effect, which is small. The $\operatorname{Im}\left\{\hat{K}_{2}+\hat{K}_{3}\right\}$ however, is much larger than that for the UK pipe system. This results in a large $\operatorname{Im}\left\{\hat{K}_{\text {total }}\right\}$, but this does not result in larger wave attenuation in $\mathrm{dB} / \mathrm{m}$ as can be seen in Fig. 4. The reasons for this are subtle and can be investigated by rewriting Eq. (3) in an alternative way as

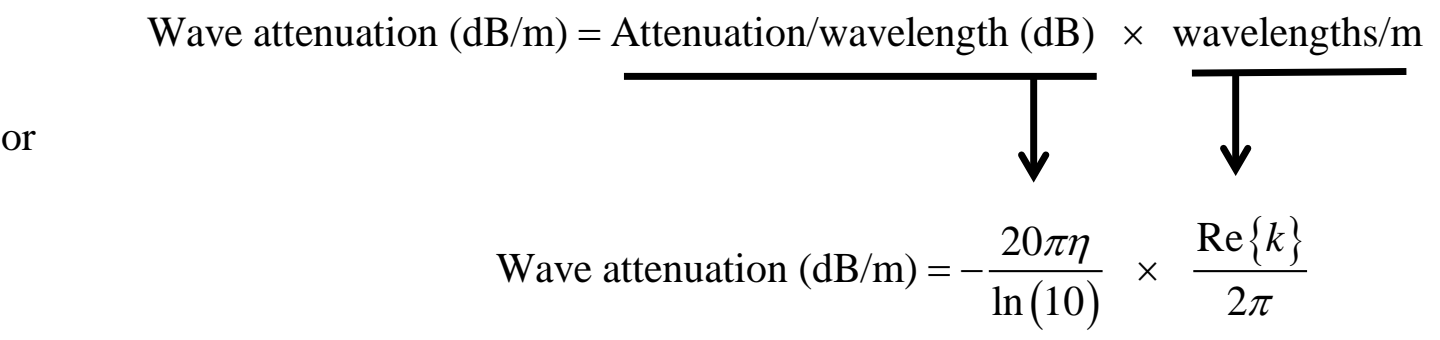

\footnotetext{
${ }^{1}$ Careful inspection of Fig. 5b(ii), shows that the imaginary part of the pipe dynamic stiffness related to the dilatational wave (which is evanescent in this case) becomes negative at high frequencies. Although this is small, it means that energy passes from the soil to the pipe in this frequency range. It occurs because of decaying wavefields in the both the pipe and the soil, with the decay being greater in the pipe than in the soil at any axial position $y$.
} 
in which $\eta=2 \operatorname{Im}\{k\} / \operatorname{Re}\{k\}$ is the effective loss factor of the pipe and the soil ${ }^{2}$. The loss factor is also related to the dynamic stiffnesses of the system and is given by $\eta=\operatorname{Im}\left\{K_{\text {total }}\right\} / \operatorname{Re}\left\{K_{\text {total }}\right\}$. From Eq. (9), it can be seen that the attenuation per wavelength is simply a function of the effective loss factor. Thus, it is possible for the soil to have no impact on the attenuation per wavelength, i.e., it does not change the effective loss factor. This could occur, for example, if both shear and dilatational waves are cut-off so they do not propagate energy away from the pipe, and there is no damping in the soil. The second term in Eq. (9), corresponding to wavelengths per metre, is inversely proportional to the wave speed $c$, as $c=\omega / \operatorname{Re}\{k\}$. In the case where the soil adds stiffness, for example as with soil type II, the wave speed in the pipe will increase, and the number of wavelengths per metre will decrease. This would manifest itself as a reduction in the attenuation per metre along the pipe, but there would be no change in energy flow from the pipe to the soil.

The effects described above are illustrated for the UK and Brazil pipe test rigs in Fig. 7 by plotting the component parts of Eq. (9). It can be seen that the mechanisms of attenuation in the pipe differ from the UK to the Brazil test rigs. In the UK system, the pipe wave-speed is not affected greatly by the soil as illustrated in the wavelength per metre plot, so the greatest influence on the wave attenuation per metre is simply the loss factor of the soil. However, in the Brazil system, the wave speed is increased by a factor of approximately two, due to the soil stiffness (observed as a reduction in wavelength/metre), and this has the effect of counteracting (to some extent at least) the additional damping added by the soil.

\footnotetext{
2 The reason for this can be best seen by noting that the wave of interest in the pipe is a longitudinal wave, and for a simple rod of Young's modulus $E$, density $\rho$ and loss factor $\eta$, the wavenumber is given by $k=\omega \sqrt{\rho / E(1+j \eta)}$. For a small loss factor such that $\eta<<1$, this becomes $k \approx \omega \sqrt{\rho / E}(1-j \eta / 2)$, so $\operatorname{Im}\{k\} / \operatorname{Re}\{k\} \approx \eta / 2$.
} 
An illustration of the way in which soil affects the wave propagation in the pipe for the UK and Brazilian pipe systems, contour plots, which depict acoustic pressure in the pipe and displacement in the soil, are shown in Fig. 8 for discrete frequencies of $200 \mathrm{~Hz}, 400 \mathrm{~Hz}$ and $600 \mathrm{~Hz}$. These plots were obtained by using the FE model described in Section 2. It can be seen that the wavelengths, corresponding to the distance between adjacent maxima (dark red) or minima (dark blue) in the pipe axial direction, are larger in the Brazilian system compared to the UK system. Furthermore, the additional energy leakage into the soil from the UK pipe system compared to the Brazilian system is clearly evident in Fig. 8, as is the localisation of acoustic energy close to the leak at higher frequencies.

\subsection{General effects of soil properties}

As discussed previously, the shear stiffness property of the soil has the most profound effect on the wave-speed in the pipe, along with the hoop stiffness of the pipe. The wave attenuation, however, is influenced by more factors and some of these effects are subtle. These are further discussed in this section to clarify the mechanisms of energy loss. The expressions for the dilatational and shear wave speeds given by Eqs. $(8 a, b)$ can be written as in terms of the normalised shear stiffness of the soil, $G / B_{\text {water }}$, to give

$$
c_{d}=\sqrt{G / B_{\text {water }}} \times \sqrt{B_{\text {water }} / \rho_{\text {soil }}} \times \sqrt{B_{\text {soil }} / G+4 / 3}, \quad c_{s}=\sqrt{G / B_{w}} \times \sqrt{B_{w} / \rho_{s}}
$$

Various wave speeds are plotted in Fig. 9 as a function of $G / B_{\text {water }}$ to illustrate generally how the shear stiffness of the soil exerts its influence. The shear wave-speed is plotted, as well as the dilatational wave-speed for the two soil types. For the UK pipe test rig (Soil type I) $G / B_{\text {water }} \approx 0.01$ and- for the Brazil pipe test rig (Soil type II) $G / B_{\text {water }} \approx 0.11$, and these are 
marked on the figure. Using Eq. (7), the pipe wave-speeds are plotted for the two systems as the shear stiffness is varied. Also plotted in Fig. 9, is the minimum possible dilatation wavespeed (labelled Min. Dil. Wave), using the values of the bulk modulus of the water and the density of the soil given in Tab. 2. The minimum possible dilatational wave speed is determined by restricting the Poisson's ratio in the soil to be non-negative. When it is set to zero then $B_{\text {soil }} / G=2 / 3$. Examination of Eq. (2a) shows that this condition results in $\operatorname{Re}\left\{K_{1}\right\}=0$. Substituting $B_{\text {soil }} / G=2 / 3$ into the expression for the dilatational wave speed, results in $c_{d}=\sqrt{2} c_{s}$.

Two specific values of $G / B_{\text {water }}$ indicated in Fig. 9 relate to soil types I and II, together with the approximate wave speeds for the UK and Brazil pipe systems given in Fig. 6. Some particular observations can be made:

1. The pipe diameter and thickness have little effect on the way in which the pipe wave speed changes as the shear stiffness of the soil is increased. This is demonstrated by the fact that the solid blue line (for the Brazilian pipe) and the dashed red line (for the UK pipe have the same trend.

2. The shear wave speed is well below the speed at which leak noise propagates along the pipe (for the geometries given in Tab. 1), for all values of soil shear stiffness, and hence the shear wave propagates in all cases transporting energy into the soil, causing wave attenuation in the pipes.

3. The dilatational wave speed in the Brazilian pipe system (soil type II) is much larger than that of the UK pipe system (soil type I), and does not vary much as the shear 
stiffness of the soil changes. This is because in the Brazilian system the dilatational wave behaviour is dominated by the bulk modulus rather than the shear modulus, which is not the case in the UK pipe system. As mentioned previously, because the dilatational wave speed is greater than the pipe wave speed, this wave does not propagate away from the pipe, and only has a mass loading effect. For soil type I, the dilatational wave speed is smaller than both pipe wave speeds for all values of shear stiffness chosen. Hence this wave would transport energy away from both pipes if they were buried in soil type I.

As discussed in the previous section, the condition that determines whether a wave in the soil propagates away from the pipe or propagates in the axial direction and is evanescent in the radial direction, is the speed of the wave in the soil compared to speed of the wave in the pipe. The limiting condition is, therefore, when the speed of the wave in the soil and the pipe are equal. By setting the pipe wave speed given in Eq. (7), to be equal to the dilatational wave speed, $c_{d}=\sqrt{\left(B_{\text {soil }}+4 G / 3\right) / \rho_{\text {soil }}}$, the ratio of the bulk modulus to the shear modulus of the soil, $B_{\text {soil }} /\left.G\right|_{\text {critical }}$, can be determined for when these wave speeds are equal, and hence the condition when the dilatational wave starts to propagate away from the soil, and have a damping effect on the pipe can be determined. The resulting equation is

$$
\left.\frac{B_{\text {soil }}}{G}\right|_{\text {critical }}=\left(\frac{1}{1+\frac{1}{\frac{E h}{2 B_{\text {water }} a}+\frac{G}{B_{\text {water }}}}}\right)\left(\frac{c_{\text {water }}}{c_{s}}\right)^{2}-\frac{4}{3}
$$


Inserting the parameters for the UK pipe and the Brazil pipe systems results in $B_{\text {soil }} /\left.G\right|_{\text {critical(UK) }}=12.75$ and $B_{\text {soil }} /\left.G\right|_{\text {critical(Brazil) }}=1.095$. This gives an upper bound on the ratio $B_{\text {soil }} / G$ for the dilatational waves to propagate and extract energy from the pipe. The lower bound on the ratio is $B_{\text {soil }} / G=2 / 3$ as discussed previously. Thus, the ranges of $B_{\text {soil }} / G$ over which a dilatational wave will propagate are given by

$$
\begin{aligned}
& \frac{2}{3} \leq \frac{B_{\text {soil }}}{G}<12.75 \quad \text { (UK pipe system, soil type I) } \\
& \frac{2}{3} \leq \frac{B_{\text {soil }}}{G}<1.095 \quad \text { (Brazil pipe system, soil type II) }
\end{aligned}
$$

Note that the lower bound is defined by the soil physics only, but the upper bound is dependent on the pipe properties as well. It can be seen that the range of $B_{\text {soil }} / G$ is quite large for the UK pipe system but is very small for the Brazil pipe system. It is evident that, as the shear stiffness of the soil increases, the range of bulk modulus for which a dilatational wave propagates away from the pipe diminishes.

To investigate the sensitivity of the wave attenuation to changes in the bulk modulus for the two systems considered in this paper, it is increased by a factor of 20 for the UK system so that $B_{\text {soil }} / G$ changes from 2.65 to 53 , and reduced by a factor 20 for the Brazil system so that $B_{\text {soil }} / G$ changes from 18.67 to 0.94 . These values are given in Tab. 4 along with other pertinent parameter values. Using a similar format to Fig. 7, the resulting attenuation of the pipe waves are shown in Fig. 10 for the modified soil properties. The attenuation of the pipe waves for the original soil properties is also shown for comparison. For the UK pipe system, it 
can be seen that when the bulk modulus is increased, there is a reduction in attenuation per wavelength as frequency increases. This is because the dilatational wave no longer propagates away from the pipe. However, it can also be seen that this effect is marginally offset because the wave speed reduces due to a small increase in mass loading, which decreases the wavelength for a given frequency, and hence increases the number of wavelengths per metre. The net effect is a large reduction in the attenuation rate compared with the original bulk modulus for the pipe wave as frequency increases. This effect is in stark contrast with the Brazil pipe system which shows that the reduction in the bulk modulus has a negligibly small effect on the wave attenuation.

\section{Conclusions}

This paper has discussed the way in which soil properties affect the behaviour of the predominantly fluid wave in buried plastic water pipes. Of particular interest, was the speed of wave propagation in the pipe and the attenuation of this wave, as these properties are important from the point of view of leak detection. The investigation has involved analytical modelling, numerical modelling, and experimental work on two very different sites corresponding to one in the UK and one in Brazil.

As the two measurement sites lead to very different vibroacoustic behaviour, an analytical investigation was carried using the analytical expression for the pipe wavenumber. The various effects of the pipe system appear in the wavenumber expression in the form of the dynamic stiffness of the pipe, the water inside the pipe, and the dilatational and shear waves in the soil. In terms of the pipe, it was found that the hoop stiffness of the pipe (as opposed to the stiffness of the water inside the pipe) mostly controls the wave speed in the pipe. In terms of the soil properties, it was found that the shear stiffness rather than the compressional stiffness of the 
soil, has the main effect on the wave-speed in the pipe. If this is large, as in the site in Brazil, this can have a dramatic effect on the wave speed; if not, as in the site in the UK, then it only has a marginal effect.

The wave attenuation in the pipe was found to be affected by the soil properties in a more complicated manner. This was investigated by examining the real and imaginary parts of the wavenumber of the pipe, and the real and imaginary parts of the dynamic stiffness of the various elements of the system. The attenuation per wavelength along the pipe is governed by five parameters: the material loss factor in the pipe wall; the loss factors associated with the bulk and shear moduli of the soil; and whether the pipe excites dilatational and/or shear waves that propagate away from the pipe. The relative importance of these properties vary from case to case. Both in the Brazil and the UK systems, it was found that the loss factor associated with the bulk modulus of the soil had a negligible effect. At low frequencies, the loss factor in the pipe has an important effect, but at higher frequencies, the shear loss factor has an important effect in the Brazil system but has a negligible effect in the UK system. Perhaps the most important physical effect, however, is whether waves in the soil propagate away from the pipe, and this is dependent upon the relative wave speeds in the soil compared to the pipe. In the UK system, both the dilatational and shear waves propagate, but in the Brazil system only the shear wave propagates; this has a profound effect on wave attenuation in the pipe. The other important effect on wave attenuation in the pipe is whether the loading effect on the pipe slows down or speeds up the wave. A stiffening effect, which such as that due to the shear wave in the soil, will increase the wave speed in the pipe, resulting in an increase the wavelength for a given frequency, reducing the attenuation per unit length. A mass loading effect, such as that due to the dilatational wave has the opposite effect. 


\section{Acknowledgements}

The authors would like to acknowledge the financial support from FAPESP, Process Nos. 2013/50412-3 and 2015/50312-4. and from EPSRC (under grant EP/K021699/1).

\section{References}

[1] B. Kingdom, Liemberger, O. Marin, The Challenge of Reducing Non-Revenue Water (NRW) in Developing Countries, Water Supply and Sanitation Board Discussion Paper Series, Paper No. 8, The World Bank, Washington, 2006.

[2] S.B. Grant, J.D. Saphores, D.L. Feldman, A.J. Hamilton, T.D. Fletcher, P.L.M. Cook, M. Stewardson, B.F. Sanders, L.A. Levin, R.F. Ambrose, A. Deletic, R. Brown, S.C. Jiang, D. Rosso, W.J. Cooper, I. Marusic, Taking the "Waste” out of "Wastewater" for Human Water Security and Ecosystem Sustainability. Science, 337, (2012), 681-686.

[3] R. Puust, Z. Kapelan, D.A. Savic, T. Koppel, A Review of Methods for Leakage Management in Pipe Networks, Urban Water Journal, Vol. 7(1), (2015) 25-45.

[4] H.V. Fuchs, R. Riehle, Ten Years of Experience with Leak Detection by Acoustic Signal Analysis, Applied Acoustics Vol. 33, (1991), 1-19.

[5] O. Hunaidi, W.T. Chu, Acoustical Characteristics of Leak Signals in Plastic Water Distribution Pipes. Applied Acoustics, Vol. 58(3), (1999), 235-254. 
[6] O. Hunaidi, W. Chu, A. Wang, W. Guan, Detecting Leaks in Plastic Pipes, Journal of the American Water Works Association, Vol. 92, (2002), 82-94.

[7] Y. Gao, M.J. Brennan, P.F. Joseph, J.M. Muggleton, O. Hunaidi, A Model of the Correlation of Leak Noise in Buried Plastic Water Pipes. Journal of Sound and Vibration. Vol. 277, (2004), 133-148.

[8] J.M. Muggleton, M.J. Brennan, R.J. Pinnington, Wavenumber Prediction in Buried Pipes for Water Leak Detection. Journal of Sound and Vibration, Vol. 249(5), (2002), 939-954.

[9] J.M. Muggleton, M.J. Brennan, P.W. Linford, Axisymmetric Wave Propagation in FluidFilled Pipes: Measurements in in-vacuo and Buried Pipes. Journal of Sound and Vibration, Vol. 270, (2004), 171-190.

[10] J.M. Muggleton, J. Yan, Wavenumber Prediction and Measurement for Buried FluidFilled pipes: Inclusion of Shear Coupling at a Lubricated Pipe/Soil Interface. Journal of Sound and Vibration, Vol. 332 (5), (2013), 1216-1230.

[11] F.C.L. Almeida, M.J. Brennan, P.F. Joseph, S. Whitfield, S. Dray, A.T. Paschoalini, On the Acoustic Filtering of the Pipe and Sensor in a Buried Plastic Water Pipe and its Effect on Leak Detection: An Experimental Investigation. Sensors, Vol. 14, (2014), 5595-5610.

[12] Y. Gao, F. Sui, J.M. Muggleton, J. Yang, Simplified Dispersion Relationships for FluidDominated Axisymmetric Wave Motion in Buried Fluid-Filled Pipes, Journal of Sound and Vibration, Vol. 375, (2016), 386-402. 
[13] F.C.L. Almeida, M.J. Brennan, P.F. Joseph, S. Dray, S. Whitfield, A.T. Paschoalini, Towards an in-situ measurement of wave velocity in buried plastic water distribution pipes for the purposes of leak location. Journal of Sound and Vibration, Vol. 359, 40-55 (2015).

[14] M.J. Brennan, M. Karimi, F.C.L. Almeida, F. Kroll de Lima, P.C. Ayala, D. Obata, A.T. Paschoalini, N. Kessissoglou. On the role of vibro-acoustics in leak detection for plastic water distribution pipes, Procedia Engineering Vol. 199 (2017), 1350-1355. 


\section{Figures}

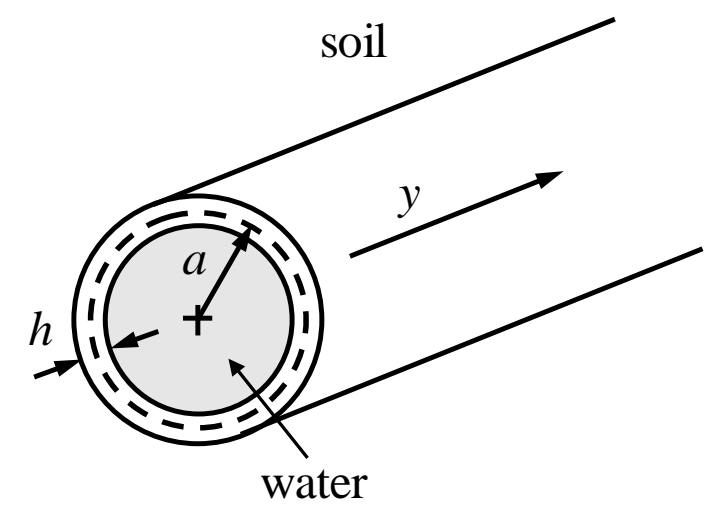

Figure 1. Schematic diagram of the buried pipe showing the pipe geometry. 


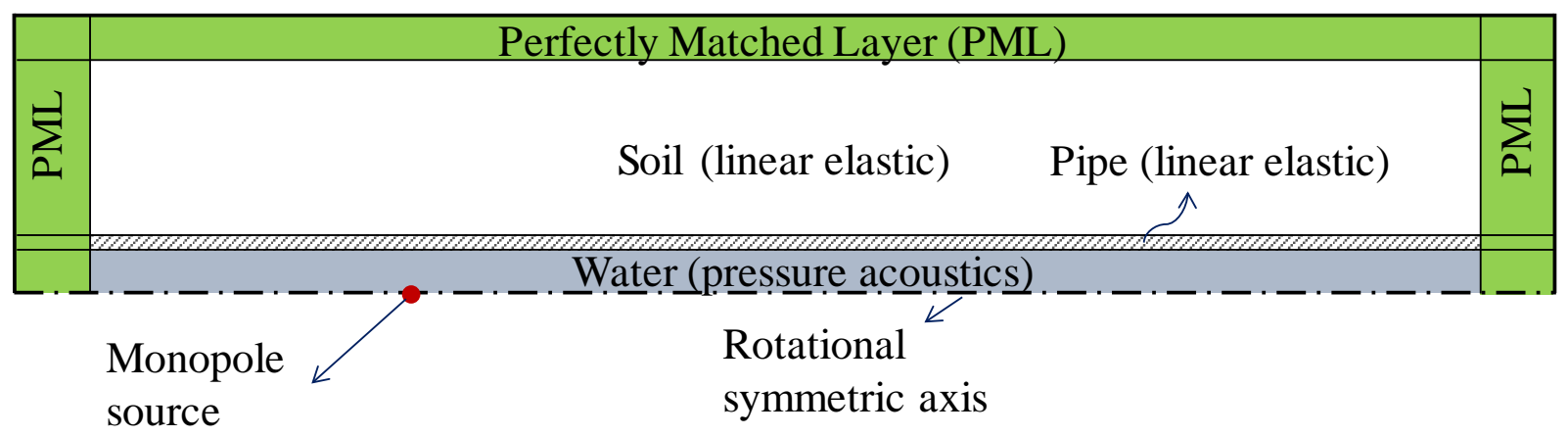

Figure 2. Finite element model of the water-pipe-soil system. 


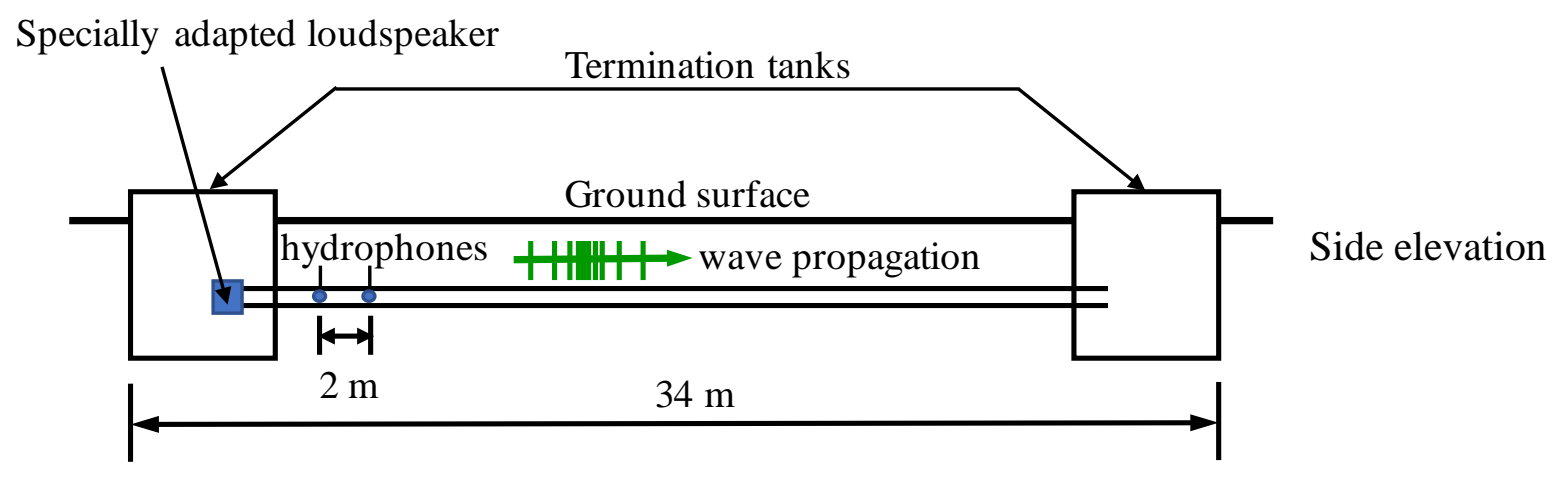

(a) UK pipe test rig with soil type I

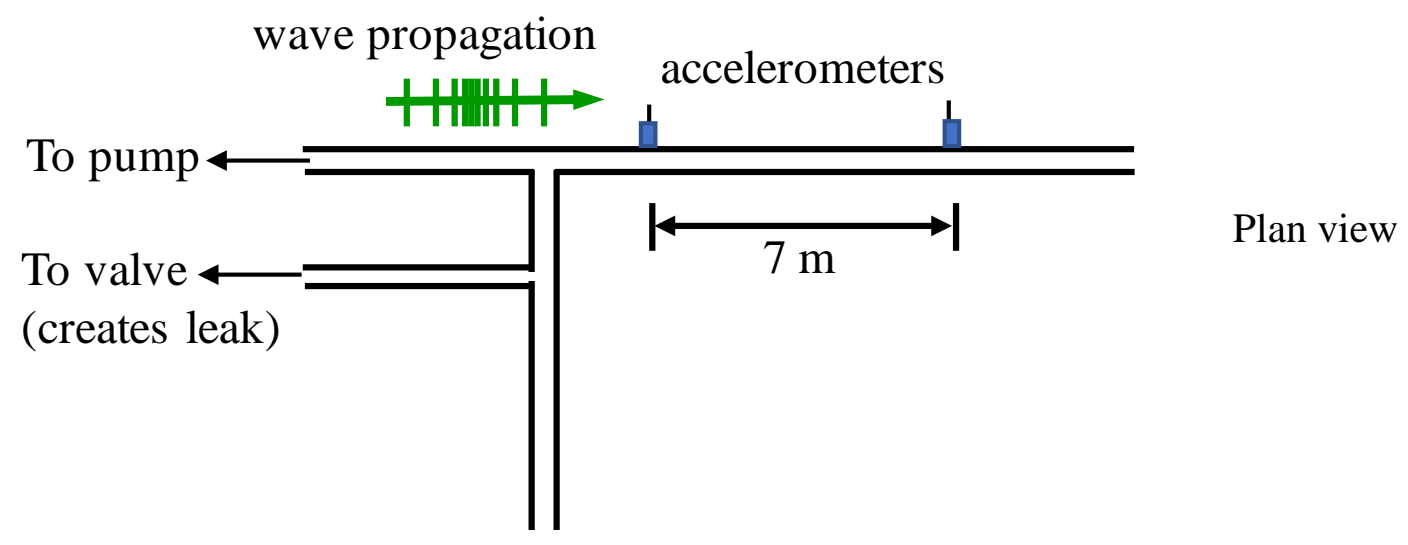

(b) Brazil pipe test rig with soil type II

Figure 3. Schematic diagrams of the two test-rigs (not to scale). (a) UK pipe test rig with soil type I, (b) Brazil pipe test rig with soil type II. The properties of the pipes are given in Table 1 and the estimated soil properties are given in Table 2. 
UK pipe test rig
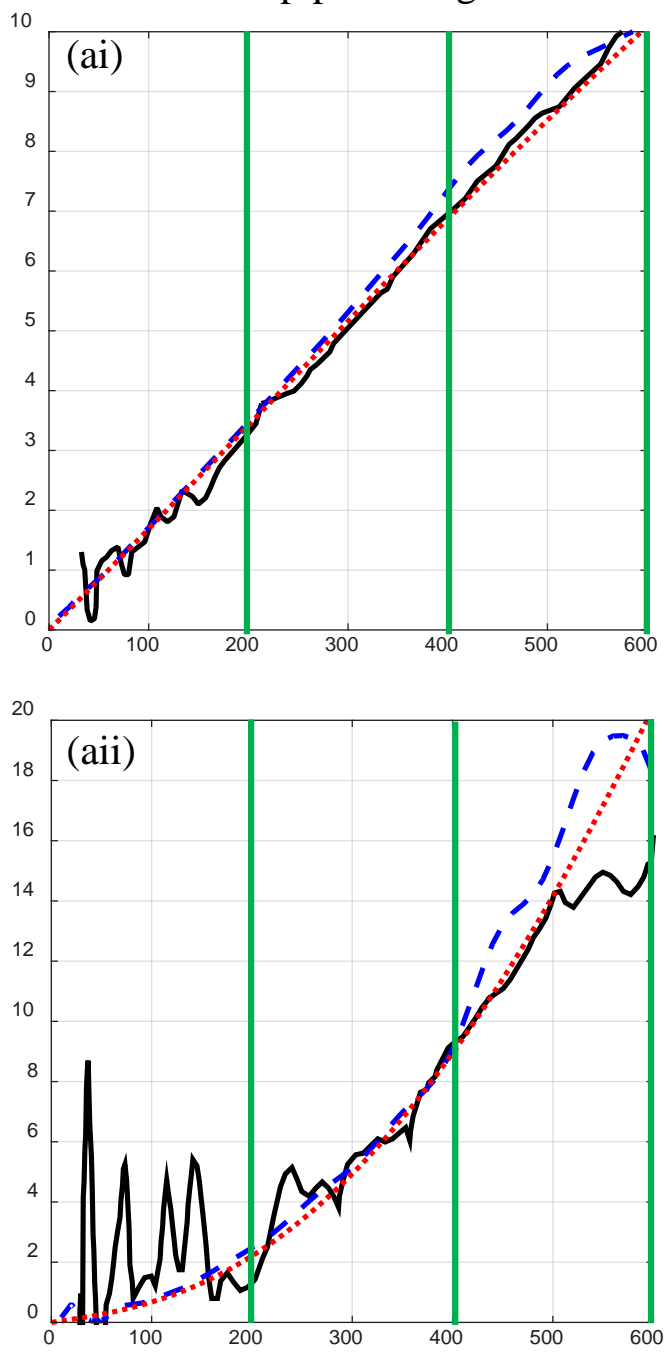

Brazil pipe test rig
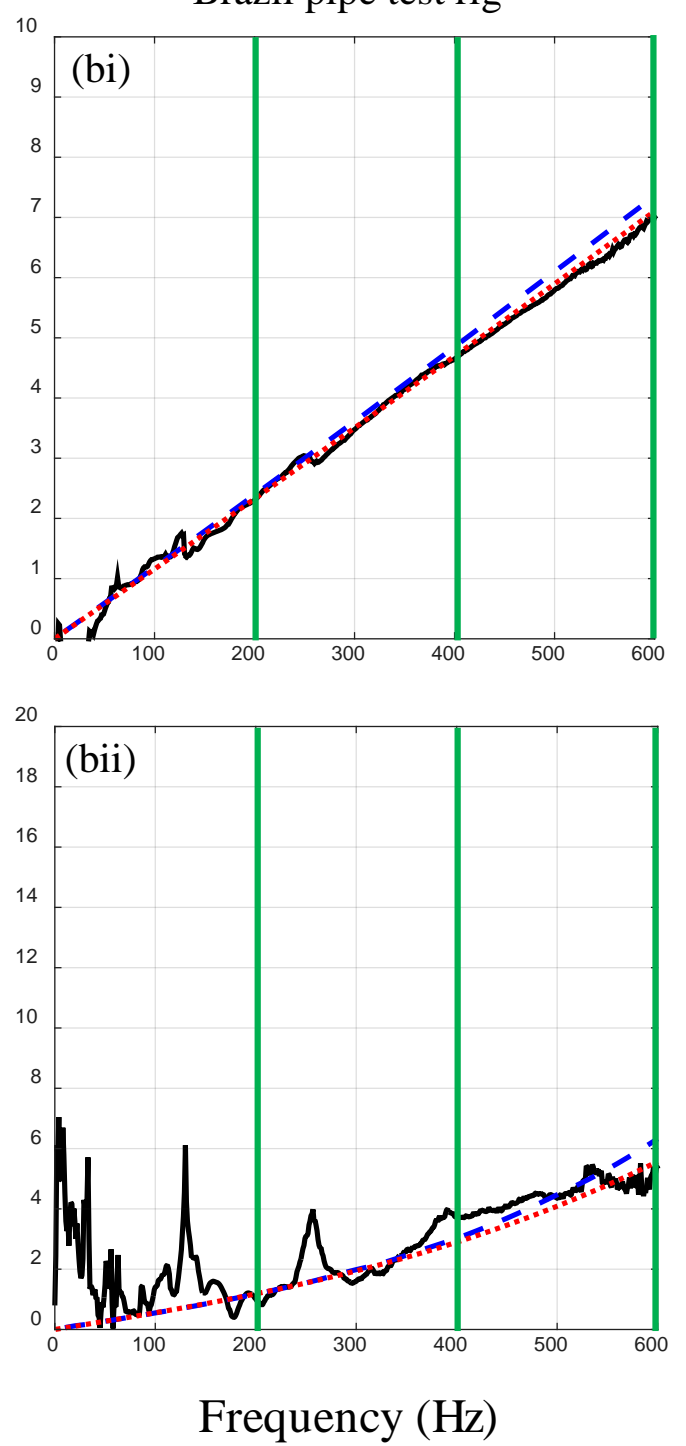

Figure 4. Measured and predicted wavenumbers, and attenuation for the two test rigs shown in Fig. 3. (a) UK pipe with soil type I, (b) Brazil pipe with soil type II. (ai) and b(i) Real part of the wave number, (aii) and (bii) Attenuation. Thick solid lines, experimental results; dotted lines, analytical results; dashed lines, numerical results. The vertical green lines indicate the frequencies where the predicted spatial vibration and acoustic field is presented in Fig. 8. 

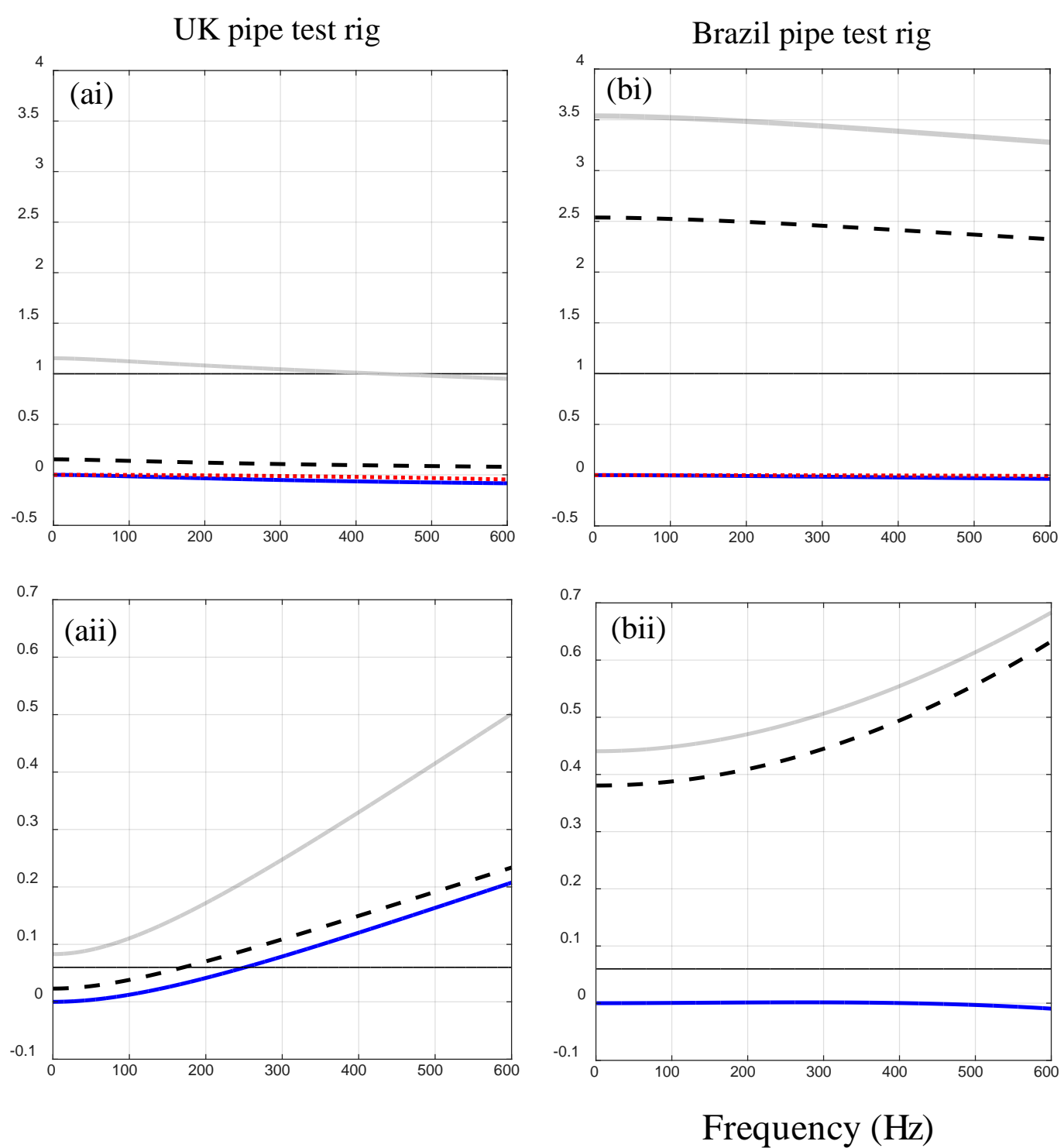

Figure 5. Normalised real and imaginary parts of the dynamic stiffness of the dilatational and shear waves for the two test rigs shown in Fig. 3. (a) UK pipe with soil type I, (b) Brazil pipe with soil type II. (ai) and b(i) Real part of the dynamic stiffness, (aii) and (bii) imaginary parts of the dynamic stiffness. $\hat{K}_{1}$ (soil dynamic stiffness representing a dilatational wave); dashed thick black line, $\hat{K}_{2}+\hat{K}_{3}$ (soil dynamic stiffness representing a shear-related wave); dotted red line, $\hat{K}_{\text {pipe }}$ (stiffness of the pipe including inertial effects); thin black line, $\hat{K}_{\text {pipe }}$ (stiffness of the pipe with no inertial effects); thick grey line, $\hat{K}_{\text {total }}$ (total dynamic stiffness). 


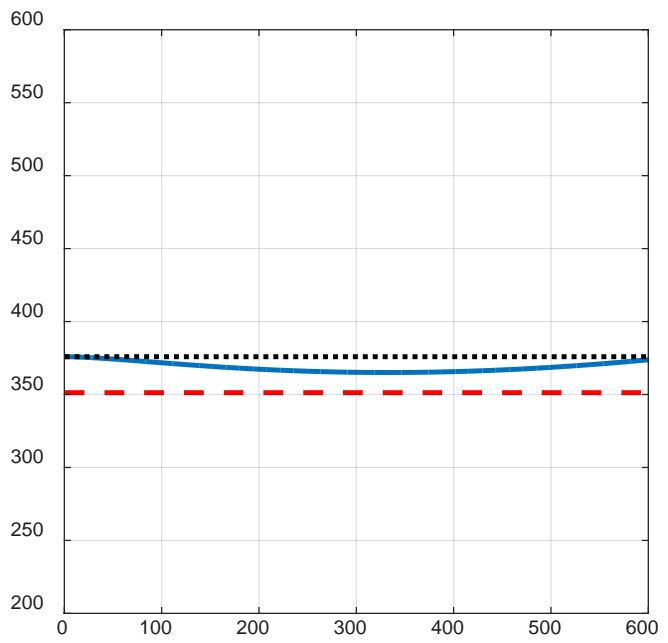

(a) UK pipe test rig with soil type I

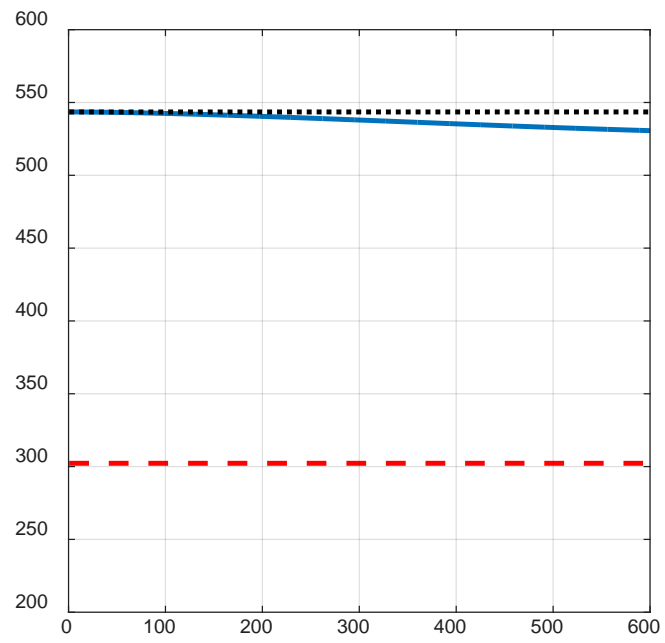

(b) Brazil pipe test rig with soil type II

Figure 6. Calculated actual and approximate wave speeds for the water-filled buried pipe. (a) UK pipe with soil type I, (b) Brazil pipe with soil type II. Solid thick blue line, actual wave speed; dotted black line, approximate wave speed given by Eq. (10); dashed red line, wave speed of the water-filled pipe alone, neglecting inertial effects. 


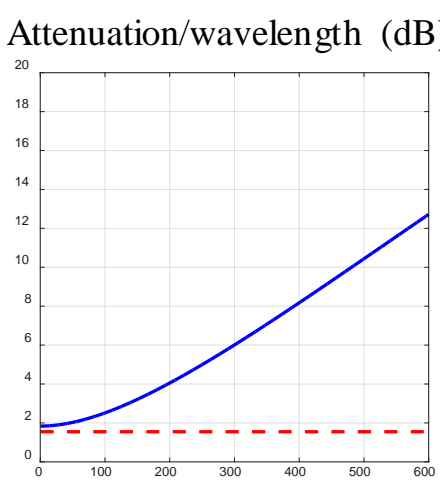

Frequency (Hz)

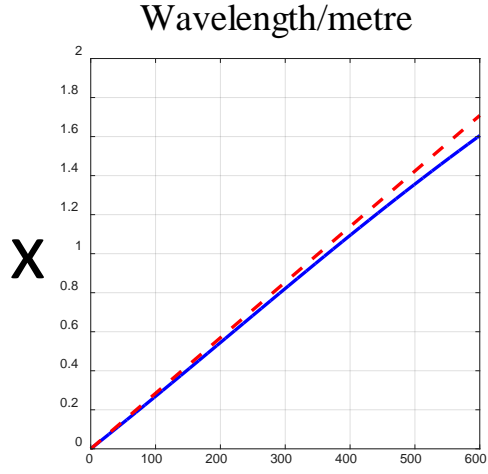

Frequency $(\mathrm{Hz})$
Attenuation (dB/metre)

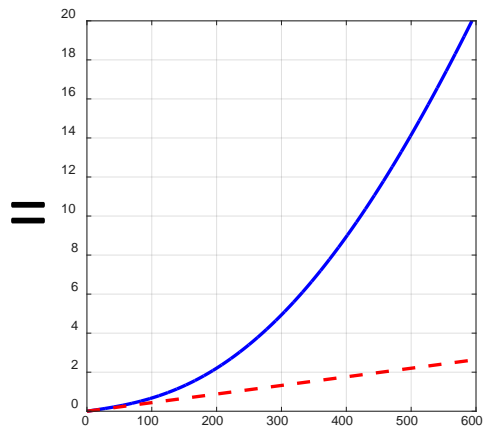

Frequency $(\mathrm{Hz})$

(a) UK pipe test rig with soil type I

Attenuation/wavelength (dB)

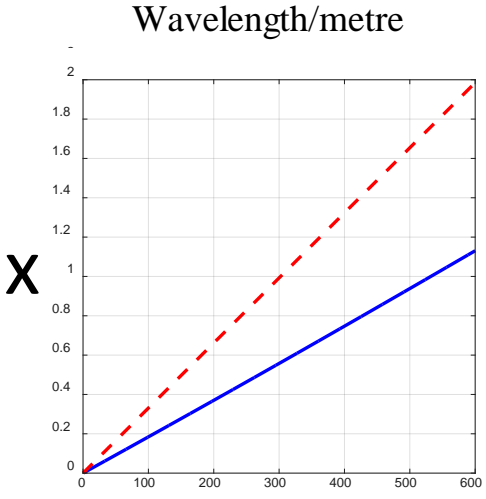

Frequency $(\mathrm{Hz})$
Attenuation (dB/metre)

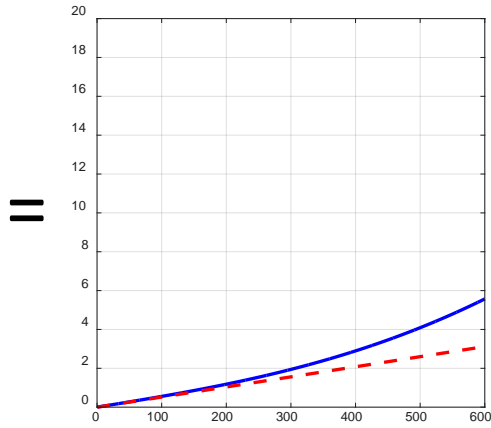

Frequency (Hz)

(b) Brazil pipe test rig with soil type II

Figure 7. Plots to show the effects of the soil on the measured attenuation in terms of $\mathrm{dB} /$ metre. The top set of graphs correspond to the UK pipe with soil type I, and the bottom set of graphs correspond to the Brazil pipe with soil type II. Solid blue lines, buried water-filled pipe; dashed red lines, pipes with no surrounding soil. 

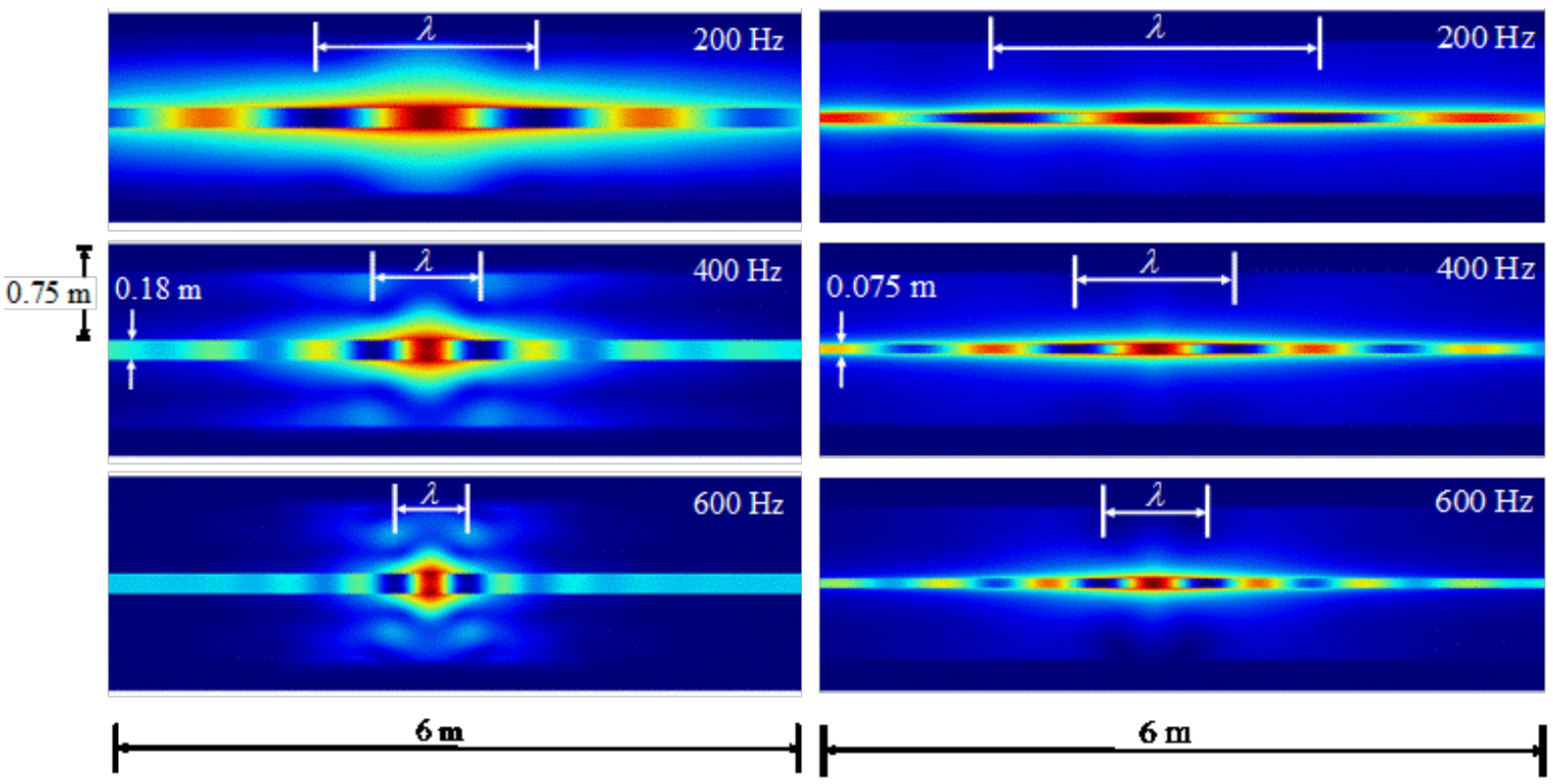

$6 \mathrm{~m}$

(a) UK pipe test rig with soil type I

(b) Brazil pipe test rig with soil type II

Figure 8. Acoustic pressure inside the pipe and displacement of the soil at three discrete frequencies corresponding to $200 \mathrm{~Hz}, 400 \mathrm{~Hz}$ and $600 \mathrm{~Hz}$, for (a) UK pipe test rig with soil type I, (b) Brazil pipe test rig with soil type II. $\lambda$ is the wavelength of the predominantly fluidwave in the pipe. 


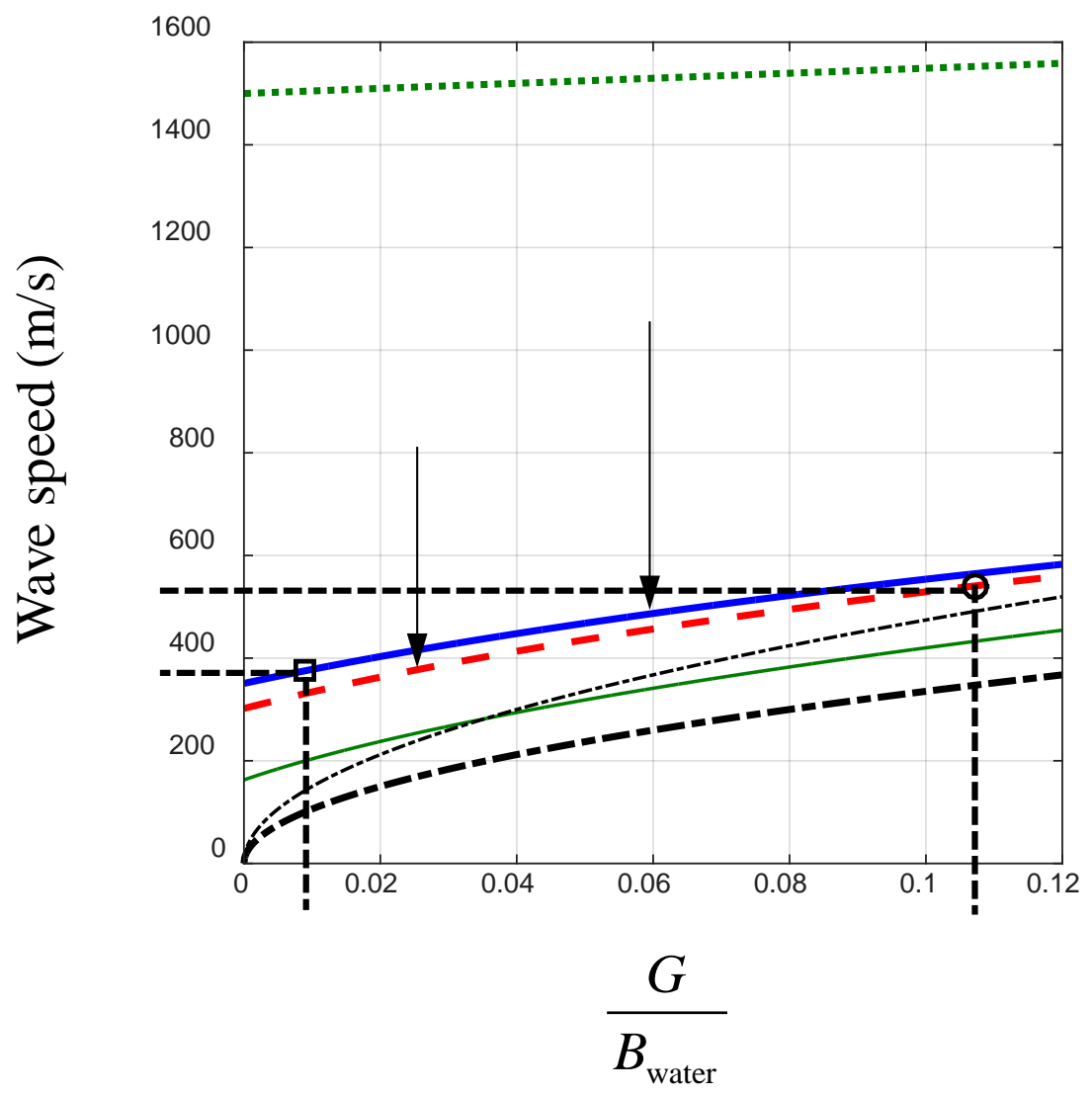

Figure 9. Wave speeds for the system as a function of the normalised shear stiffness of the soil. The minimum dilatational wave speed is for a Poisson's ratio of zero in the soil. 

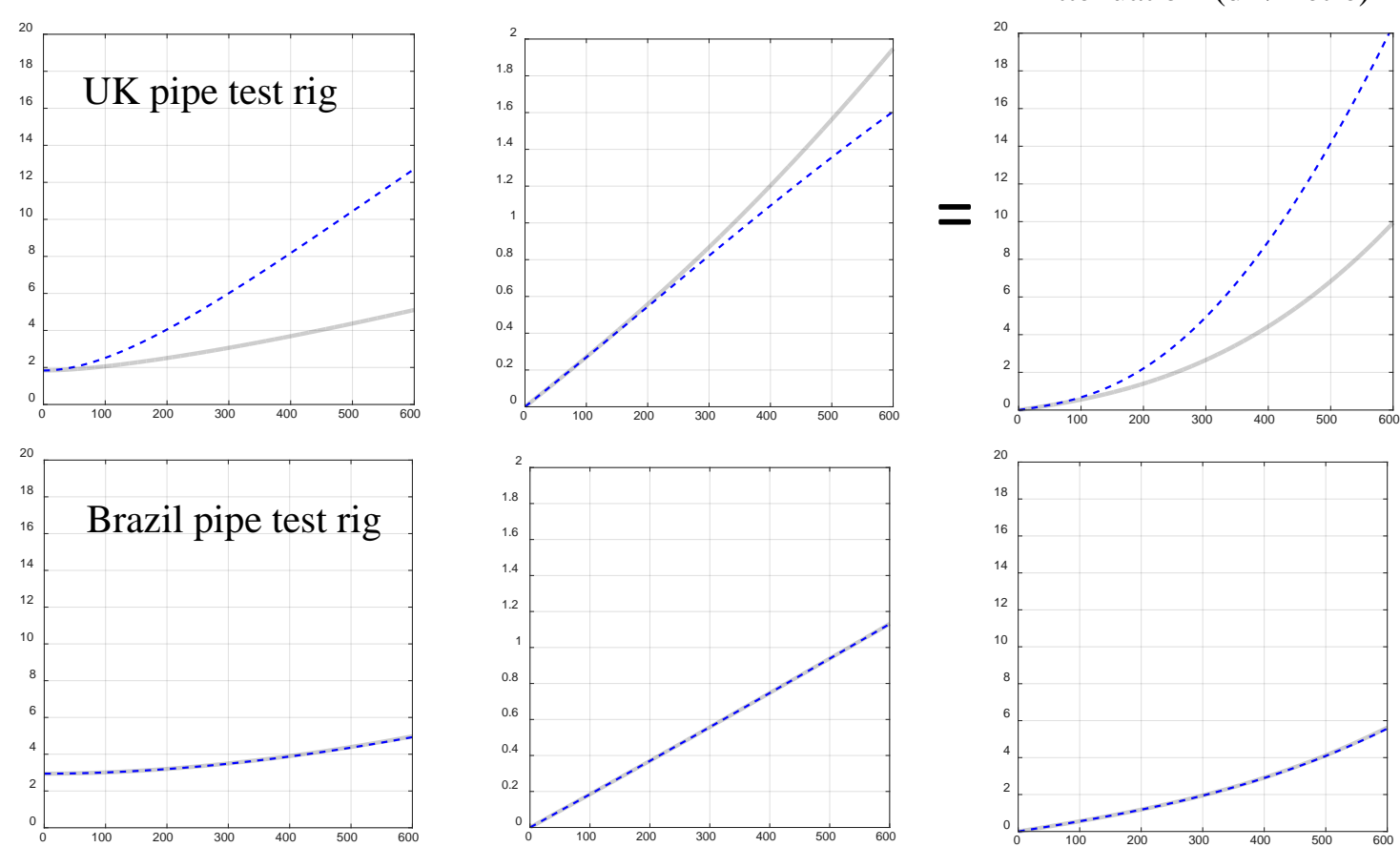

Figure 10. Plots to show the effects of the modified soil properties given in Tab. 4 on the measured attenuation in terms of $\mathrm{dB} /$ metre. The top set of graphs correspond to the UK pipe with soil type I, and the bottom set of graphs correspond to the Brazil pipe with soil type II. Dashed blue line, original soil properties; solid grey line modified soil properties. 


\section{Tables}

\begin{tabular}{c|c|c}
\hline Properties & UK & Brazil \\
\hline Young's modulus, $E\left(\mathrm{~N} / \mathrm{m}^{2}\right)$ & $2 \times 10^{9}$ & $2 \times 10^{9}$ \\
Density $\rho_{p}\left(\mathrm{~kg} / \mathrm{m}^{3}\right)$ & 900 & 900 \\
Loss factor $\eta_{p}$ & 0.06 & 0.06 \\
Pipe radius $(\mathrm{mm})$ & 84.5 & 35.8 \\
Pipe-wall thickness $(\mathrm{mm})$ & 11 & 3.4 \\
\hline
\end{tabular}

Table 1. Pipe Properties

\begin{tabular}{c|c|c|c}
\hline Properties & $\begin{array}{c}\text { Soil type I } \\
(\mathrm{UK})\end{array}$ & $\begin{array}{c}\text { Soil type II } \\
(\text { Brazil) }\end{array}$ & Water \\
\hline Bulk modulus $B_{\text {soil, } w}\left(\mathrm{~N} / \mathrm{m}^{2}\right)$ & $5.3 \times 10^{7}$ & $4.5 \times 10^{9}$ & $2.25 \times 10^{9}$ \\
Shear modulus, $G\left(\mathrm{~N} / \mathrm{m}^{2}\right)$ & $2.0 \times 10^{7}$ & $2.41 \times 10^{8}$ & \\
Bulk and shear loss factor $\eta_{d, s}$ & 0.15 & 0.15 & \\
Density $\rho_{\text {soil }}\left(\mathrm{kg} / \mathrm{m}^{3}\right)$ & 2000 & 2000 & \\
\hline
\end{tabular}

Table 2. Soil and Water Properties

\begin{tabular}{c|c|c|c|c|c|c}
\hline & \multicolumn{2}{|c|}{ Soil type I } & \multicolumn{2}{c|}{ Soil type II } & \multicolumn{2}{c}{ Buried pipe } \\
\cline { 2 - 7 } & Dilatational & Shear & Dilatational & Shear & UK & Brazil \\
& $c_{d}$ & $c_{s}$ & $c_{d}$ & $c_{s}$ & $c$ & $c$ \\
\hline Soil Wave speeds $(\mathrm{m} / \mathrm{s})$ & 200 & 100 & 1553 & 347 & 376 & 544 \\
Angle of propagation $\left(^{\circ}\right)$ & 58 & 75 & - & 50 & 0 & 0 \\
\hline
\end{tabular}

Table 3. Wave properties of the soils and buried pipes 


\begin{tabular}{|c|c|c|c|c|}
\hline & \multicolumn{2}{|c|}{$\begin{array}{l}\text { UK pipe system } \\
\text { Soil type I }\end{array}$} & \multicolumn{2}{|c|}{$\begin{array}{l}\text { Brazil pipe system } \\
\text { Soil type II }\end{array}$} \\
\hline & $\begin{array}{c}\text { Original } \\
\text { system }\end{array}$ & $\begin{array}{l}\text { Modified } \\
\text { system }\end{array}$ & $\begin{array}{c}\text { Original } \\
\text { system }\end{array}$ & $\begin{array}{l}\text { Modified } \\
\text { system }\end{array}$ \\
\hline Bulk modulus $B_{s}\left(\mathrm{~N} / \mathrm{m}^{2}\right)$ & $5.3 \times 10^{7}$ & $1.06 \times 10^{9}$ & $4.5 \times 10^{9}$ & $2.25 \times 10^{8}$ \\
\hline Shear modulus $G_{s}\left(\mathrm{~N} / \mathrm{m}^{2}\right)$ & \multicolumn{2}{|c|}{$2.0 \times 10^{7}$} & \multicolumn{2}{|c|}{$2.41 \times 10^{8}$} \\
\hline $\begin{array}{c}B_{s} / G \\
\text { Shear wave speed }(\mathrm{m} / \mathrm{s})\end{array}$ & \multicolumn{2}{|c|}{100} & \multicolumn{2}{|c|}{347} \\
\hline $\begin{array}{l}\text { Dilatational wave speed } \\
(\mathrm{m} / \mathrm{s})\end{array}$ & 200 & 739 & 1553 & 524 \\
\hline $\begin{array}{l}\text { Dilatational wave } \\
\text { propagates }\end{array}$ & Yes & No & No & Yes \\
\hline $\begin{array}{l}\text { Limits for dilatational } \\
\text { wave propagation }\end{array}$ & \multicolumn{2}{|c|}{$\frac{2}{3} \leq \frac{B_{s}}{G}<12.75$} & \multicolumn{2}{|c|}{$\frac{2}{3} \leq \frac{B_{s}}{G}<1.095$} \\
\hline
\end{tabular}

Table 4. Properties of the systems for the parametric study 